\title{
QUEEN'S
UNIVERSITY
BELFAST
}

\section{Work Ethic, Social Ethic, no Ethic: Measuring the Economic Values of Modern Christians}

Colvin, C. L., \& McCracken, M. (2017). Work Ethic, Social Ethic, no Ethic: Measuring the Economic Values of Modern Christians. Journal of Applied Econometrics, 32(5), 1043-1053. https://doi.org/10.1002/jae.2543

Published in:

Journal of Applied Econometrics

Document Version:

Peer reviewed version

Queen's University Belfast - Research Portal:

Link to publication record in Queen's University Belfast Research Portal

Publisher rights

Copyright $\odot 2016$ John Wiley \& Sons, Ltd.

This is the peer reviewed version of the following article: Colvin, C. L., and McCracken, M. (2016) Work Ethic, Social Ethic, no Ethic: Measuring the Economic Values of Modern Christians. J. Appl. Econ, which has been published in final form at

$\mathrm{http}: / /$ onlinelibrary.wiley.com/wol1/doi/10.1002/jae.2543/abstract. This article may be used for non-commercial purposes in accordance with

Wiley Terms and Conditions for Self-Archiving.

\section{General rights}

Copyright for the publications made accessible via the Queen's University Belfast Research Portal is retained by the author(s) and / or other copyright owners and it is a condition of accessing these publications that users recognise and abide by the legal requirements associated with these rights.

Take down policy

The Research Portal is Queen's institutional repository that provides access to Queen's research output. Every effort has been made to ensure that content in the Research Portal does not infringe any person's rights, or applicable UK laws. If you discover content in the Research Portal that you believe breaches copyright or violates any law, please contact openaccess@qub.ac.uk. 


\title{
Work Ethic, Social Ethic, No Ethic: Measuring the Economic Values of Modern Christians*
}

\author{
Christopher L. Colvin ${ }^{\dagger} \quad$ Matthew McCracken \\ Queen's University Belfast
}

July 2016

\begin{abstract}
Benito Arruñada finds evidence of a distinct Protestant social ethic in the ISSP's 1998 Religion II Survey (Economic Journal 2010; 120: 890-918). We replicate Arruñada's results using his broad definition of Protestantism and our new narrow definition, which includes only those ascetic denominations that Max Weber singled out for possessing a strong capitalist work ethic. We then extend this analysis to the ISSP's 2008 Religion III Survey, the most recent comparable international questionnaire on religious attitudes and religious change. We find no evidence of a Calvinist work ethic, and suggest that Arruñada's Protestant social ethic continues into the twenty-first century.

JEL Codes: J24, Z12.
\end{abstract}

Keywords: work ethic, social ethic, international social survey programme, replication.

${ }^{*}$ We thank Matthias Blum, Alan Fernihough, Philip Fliers, Stuart Henderson, John Turner and especially an anonymous referee for comments and suggestions.

$\dagger$ Corresponding author. Address: Queen's Management School, Queen's University Belfast, Riddel Hall, 185 Stranmillis Road, Belfast, Northern Ireland, BT9 5EE, UK. Email: chris.colvin@qub.ac.uk. Telephone: +44 (0)289097 4735 . 


\section{Introduction}

How do religious beliefs, cultural norms and personal values influence decision-making in society? Max Weber (2011) argues that Protestantism held a distinctive role in the rise of modern capitalism. Central to Weber's century-old thesis is the difference between Protestants and Catholics in terms of attitudes towards work, thrift and self-improvement. ${ }^{1}$ Recent studies have tested Weber's "work ethic" hypothesis in historical settings (e.g. Becker and Woessmann, 2010; Cantoni, 2015; Blum and Strebel, 2016), but results remain conflicted and controversial. Starting with Barro and McCleary (2003) and Guiso et al. (2003), the use of cross-country surveys to help disentangle institutional from religion-related effects has become a popular social research methodology, one which helps to address whether the economic values of Protestants and Catholics still differ today. ${ }^{2}$ Arruñada (2010) is one such study, and is particularly interesting as it tests an alternative hypothesis involving a Protestant "social ethic".

Arruñada's article, the subject of the present note, explores the idea that Protestants exert a greater effort of trust towards their Church, education system and government. ${ }^{4} \mathrm{He}$ pits this against a simplified interpretation of Weber's work ethic: Protestants are more willing to work, and to work longer hours, than their Catholic peers. He tests these hypotheses using the results of the International Social Survey Programme's (ISSP) Religion II Survey, conducted in 1998, which asked respondents located in 32 countries a series of questions useful for the social scientific study of religion. Arruñada's results indicate that Protestants indeed worked more hours than Catholics; however, after controlling for fixed country effects, this difference disappears. He argues instead that his findings are consistent with the idea that Protestants monitor one another's conduct more carefully, and support the political and legal institutions that facilitate this; Protestants promote a social rather than a work ethic.

We revisit Arruñada's findings in two ways: (1) we replicate a subset of his results in a narrow sense, to the best of our ability; and (2) we extend his analysis to 2008, the most recent year for which the ISSP has conducted its survey of religious attitudes and religious change. ${ }^{5}$ We repeat our analysis for both a strict Calvinist $^{6}$ and an all-encompassing definition of

\footnotetext{
${ }^{1}$ Weber's Protestant ethic hypothesis concerns the direct content of Protestant teachings as much as the overall Protestant lifestyle, including - in his work on Protestant sects - followers' economic interaction within exclusive social organisations (Kalberg, in: Weber, 2011).

${ }^{2}$ Social survey data have not solved the controversies: Durlauf et al. (2012) find that Barro and McCleary's (2003) results cannot be replicated with Bayesian methods, and so conclude 'there is no evidence that religious beliefs [...] have a direct robust relationship with economic growth' (p. 1074).

${ }^{3}$ Arruñada (2010) has been cited 39 times in Crossref and 143 times in Google Scholar (July 2016).

${ }^{4}$ Like La Porta et al. (1997), Arruñada's focus is on Putnam's (1993) idea that hierarchical religions such as Catholicism discourage interpersonal trust, and therefore hinder social and market interaction.

${ }^{5}$ We only replicate results which can be extended to 2008 . Where multiple replicable proxies for a hypothesis are available, we choose to replicate and extend only a subset in order to maintain brevity.

${ }^{6}$ Weber (2011) identifies Calvinists in particular as possessing a distinctive work ethic. He argues this stems from their dogma of predestination, which encourages worldly activity as a means of gaining the self-confidence to be counted among the elect rather than the reprobate.
} 
Protestantism. ${ }^{7}$ Our results, alongside Arruñada's predictions and findings, are summarised in Table $1 .^{8}$ While we conclude that the precise definition of Protestantism does not greatly influence the results for 1998, we argue that Arrunada's findings are strengthened by our analysis of the 2008 survey wave. Catholics now exert notably less confidence in their political and legal institutions than Protestants - a result Arrunada predicted, but which was not borne out in the 1998 data.

\section{Data and empirical strategy}

The ISSP 1998 survey contains 39,034 observations. Alongside the demographic characteristics of all respondents, the dataset includes questions on feelings, values, religious beliefs and practices, social habits and opinions. Like Arrunada, we drop observations on individuals with missing values and non-Christian affiliations. We adopt two definitions of Protestantism: a strict Calvinist definition that constitutes just 1,212 individuals; ${ }^{9}$ and a broader definition that includes 6,604. ${ }^{10}$ Table 2 provides summary statistics for variables defined exactly as in Arruñada (2010: 910-914) for our two Protestant definitions, ${ }^{11}$ alongside statistics taken directly from Arruñada (2010: 903-904) for comparison. ${ }^{12}$

We matched the ISSP's 2008 questions onto the 1998 set and have sufficient data to extend at least five of Arruñada's models. A full list of variable definitions across both survey waves is reported in the online supplementary materials (Table S1). Again, we adopt Calvinist $(1,262$ individuals) and broad $(9,573)$ definitions of Protestantism. Summary statistics are reported in Table 2, with the difference in means between 1998 and 2008 reported in the supplementary materials (Table S2). The proportion of respondents in work has increased by 11 percentage points, while the average number of working hours worked per week has decreased by 1.5 hours. Interestingly, while working Protestants worked a 39-hour week in 2008, their Catholic counterparts worked 40-hour weeks. The difference is starker for Calvinists. This may, of

\footnotetext{
${ }^{7}$ This broader definition is probably closest to that used in Arruñada (2010).

${ }^{8}$ We also report those results from Arruñada (2010) that we did not replicate and extend (see n. 5).

${ }^{9}$ The strict Calvinist or ascetic definition constitutes only those denominations that can trace their roots to John Calvin's teachings: Presbyterian (ISSP 1998 code 43); Congregational, excluding Slovakia (45); Free Church, excluding Norway and Denmark (48); Other Protestant State Churches, including only the Netherlands, Slovakia, Hungary and Switzerland (49); United Church, excluding Latvia (55); and Free Presbyterians (60).

10 The broad all-encompassing definition constitutes: Baptists (ISSP 1998 code 40); Methodists (41); Lutherans (42); Presbyterians (43); Anglicans (44); United Reformed Church (45); Unitarians (47); Free Church (48); Other Protestant State Churches (49); United Church (55); Free Presbyterians (60); Brethren (61); Pentecostal (62); Salvation Army (64); Seventh-day Adventists (65); Hussites (67); NonDenominational (91); and Other Christians (92).

${ }^{11}$ As in Arruñada (2010), we define Catholics as the sum of Roman Catholics (ISSP 1998 code 10) and Greek Catholics (11). While the definition of Catholicism remains identical across samples, variable standardisation means summary statistics sometimes differ.

${ }^{12}$ Arruñada (2010) counts an additional 139 Protestants and 58 Catholics. We attribute this disparity to our decision to exclude respondents hailing from Bulgaria or Canada, a choice we make because these countries were not surveyed in both 1998 and 2008.
} 
course, be due to common changes in working practices that happen to correlate with the denomination of countries' majority religions. Regression analysis is therefore necessary to control for such fixed effects.

Indeed, our empirical strategy, which is identical to Arruñada (2010), is to estimate the following equations:

$$
\begin{aligned}
& Y_{i}=\alpha_{0}+\alpha_{0 c} \text { Catholic }+\beta_{1} \text { Faith }+\beta_{2} \text { Religious upbringing }+\beta_{3} \text { Education } \\
& \left.+\beta_{1 c} \text { (Catholic } \times \text { Faith }\right) \\
& +\beta_{2 c} \text { (CatholicR } \times \text { Religious upbringing) } \\
& +\beta_{3 c}(\text { Catholic } \times \text { Education })+\sum_{t}\left(\beta_{t} \text { Control }_{m} \text { variables }\right) \\
& +\sum_{r}\left(\beta_{r} \text { Control }_{m} \text { dummies }\right)
\end{aligned}
$$

where each dependent variable, $Y_{i}$, represents a value from five variables constructed from survey questions present in both datasets: two relating to the Protestant work ethic hypothesis (Positive working hours and Working hours of workers), and three to the social ethic hypothesis (Religious practice, Trust institutions and Family importance). The coefficient $\alpha_{0 c}$ represents the difference attributed to being Catholic rather than Protestant. Faith, Upbringing and Education have been standardised and measure attitudes relative to the average person in the sample. With Protestantism as the omitted category, the interaction terms measure the differential impact of Catholic Faith, Upbringing and Education.

Our replication of Arruñada's results, using both the strict Calvinist and broader definitions of Protestantism, is reported in Table 3, Panel (a), alongside those taken from Arrunada (2010: 905) for comparison. Our extension, using the ISSP's 2008 survey wave, is reported in Panels (a) of Tables 4 and 5. As in Arruñada (2010), the overall effect of "being Catholic" is estimated in a simplified version of equation (1), in which Faith, Upbringing and Education and their interactions are excluded, but the demographic and country controls left in. The results of these exercises are reported in Panels (b) of Tables 3, 4 and 5. A comparison of residual heterogeneity between Catholics and Protestants is reported the supplementary materials (Table S3).

\section{Results and robustness}

Following Weber's work ethic hypothesis, Arruñada predicts that fewer Catholics should be in work, and should work fewer hours. His results suggest this is not the case. Our results confirm his findings for 1998 (models 1 and 2) and show they also hold for 2008 (models 6, 7, 11 and 12). A comparison of residual heterogeneity in these regressions (Table S3) suggests Calvinists have become more homogenous with respect to their working practices between 1998 and 2008.

Predictions relating to Arruñada's alternative social ethic hypothesis are in three parts: (1) Catholics exert less effort in mutual social control than Protestants; (2) they support political and legal institutions less; and (3) they hold less homogenous values. In the first (models 3, 8 and 13), measured by Arruñada as the relationship between Education and Religious practice, 
predictions match results in all cases: the difference between Catholics and Protestants in 1998 is -0.091 standard deviations for Arruñada, -0.114 for our Calvinist definition and -0.078 for all Protestants; and in 2008 it is -0.099 for Calvinists and -0.071 for all Protestants. This result suggests Catholics consistently exerted less social control than their Protestant peers. The difference in the simplified regressions in Panels (b) switches sign for all Protestants in 2008 (model 13); Catholics now practice less than Protestants. Comparing residual heterogeneity (Table S3) suggests Protestants have become less homogenous with respect to their religious practices in the decade between the two survey years.

In the second social ethic hypothesis (models 4, 9 and 14), measured here in the coefficient of Catholic in a regression of Trust institutions, our findings confirm those of Arrunada for 1998: a result that is not statistically significant. However, our results are quite different for 2008: Arruñada's original unrealised prediction of a statistically significant negative coefficient is here realised for the broader definition of Protestantism, both in Panel (a) and the simplified Panel (b); Catholics now have less confidence than Protestants in the institution of parliament and in their courts and legal system.

The third social ethic hypothesis (models 5, 10 and 15) is again read in the coefficient of Catholic, this time in a regression of Family importance. Our results are almost identical to those of Arruñada for 1998, and persist into 2008: statistically significant and positive; Catholic support for the family, defined here as their intolerance of premarital sex, remains greater than that of Protestants.

\section{Discussion and conclusion}

We have successfully replicated Arrunada (2010) in a narrow sense, extended his results to a much stricter definition of Protestantism, and shown that an analysis of the ISSP's newest religion dataset yields compatible findings. We suggest our analysis of Calvinists, the ascetic denomination to which Weber ascribed unique capitalist economic values, has strengthened Arruñada's findings. Like Protestants more generally, modern Calvinists display little evidence of having a different work ethic than their Catholic peers. But our analysis of values towards the rule of law suggests one significant change between 1998 and 2008: Protestants placed more confidence in civic institutions in 2008, a result predicted but not found by Arrunada for 1998. Understanding the reason for this temporal change should be the subject of further enquiry.

\section{References}

Arruñada, Benito. 2010. Protestants and Catholics: Similar work ethic, different social ethic. Economic Journal 120(547): 890-918. DOI: 10.1111/j.1468-0297.2009.02325.x

Barro, Robert J., and Rachel M. McCleary. 2003. Religion and economic growth across countries. American Sociological Review 68(5): 760-781. 
Becker, Sascha O. and Ludger Woessmann. 2010. Was Weber wrong? A human capital theory of Protestant economic history. The Quarterly Journal of Economics 124(2): 531-596. DOI: 10.1162/qjec.2009.124.2.531

Blum, Matthias and Matthias Strebel. 2016. Max Weber and the First World War: Protestant and Catholic living standards in Germany, 1915-1919. Journal of Institutional Economics. DOI: 10.1017/S1744137415000533

Cantoni, Davide. 2015. The economic effects of the Protestant Reformation: Testing the Weber hypothesis in the German lands. Journal of the European Economic Association 13(4): 561-598. DOI: 10.1111/jeea.12117

Durlauf, Steven N., Andros Kourtellos and Chih Ming Tan. 2012. Is God in the details? A re-examination of the role of religion in economic growth. Journal of Applied Econometrics 27(7): 1059-1075. DOI: 10.1002/jae.1245

Guiso, Luigi Paola Sapienz and Luigi Zingales. 2003. People's opium? Religion and economic attitudes. Journal of Monetary Economics 50(1): 225-282. DOI: 10.1093/oep/gpr025

La Porta, Rafael, Florencio Lopez-de-Silanes, Andrei Shleifer and Robert W. Vishny. 1997. Trust in large organizations. American Economic Review: P\&P 87(2): 333-338.

Levene, Howard. 1960. Robust tests for equality of variances. In: Ingram Olkin (ed.), Contributions to Probability and Statistics: Essays in Honor of Harold Hotelling, pp. 278-292. Stanford University Press: Stanford.

Putnam, Robert. 1993. Making Democracy Work: Civic Traditions in Modern Italy. Princeton University Press: Princeton.

Weber, Max. 2011 [1920]. The Protestant Ethic and the Spirit of Capitalism. Translated and introduced by Stephen Kalberg. OUP: New York.

\section{Data sources}

International Social Survey Programme 1998: Religion II (ISSP 1998). Includes codebook. Available at: http://www.issp.org/.

International Social Survey Programme 2008: Religion III (ISSP 2008). Includes codebook. Available at: http://www.issp.org/. 
Table 1: Summary of predictions and results, including those not replicated and extended in this study

\begin{tabular}{|c|c|c|c|c|c|c|c|c|c|c|}
\hline \multirow[t]{4}{*}{$\begin{array}{l}\text { Hypotheses } \\
\end{array}$} & \multirow[t]{4}{*}{$\begin{array}{l}\text { Variables and tests } \\
\text { na }\end{array}$} & \multirow[t]{4}{*}{ Proxies used } & \multirow{4}{*}{$\begin{array}{l}\text { Available for } \\
1998 \text { and } 2008 ?\end{array}$} & \multirow{4}{*}{$\begin{array}{l}\text { Included in } \\
\text { analysis? }\end{array}$} & \multicolumn{6}{|c|}{ 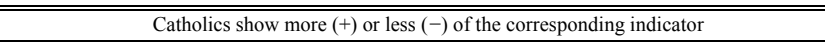 } \\
\hline & & & & & \multirow[t]{3}{*}{ Predictions } & \multicolumn{5}{|c|}{ Results } \\
\hline & & & & & & \multirow[t]{2}{*}{ Arruñada } & \multicolumn{2}{|c|}{ ISSP 1998} & \multicolumn{2}{|c|}{ ISSP 2008} \\
\hline & & & & & & & Calvinist & Broad & Calvinist & Broad \\
\hline \multicolumn{11}{|l|}{ Work ethic: } \\
\hline \multirow{4}{*}{$\begin{array}{l}\text { Catholics work less and less effectively } \\
\text { than Protestants }\end{array}$} & \multirow[t]{3}{*}{ Willingness to work and work effort } & Working hours & Yes & No & - & n. s. & & & & \\
\hline & & Positive working hours & Yes & Yes & - & n. s. & n. s. & n. s. & n. s. & n. s. \\
\hline & & Working hours of those working & Yes & Yes & - & n. s. & n. s. & n. s. & n. s. & n. s. \\
\hline & Earthly achievements & Success index & No & No & - & n. s. & & & & \\
\hline \multicolumn{11}{|l|}{ Social control: } \\
\hline \multirow{4}{*}{$\begin{array}{l}\text { Catholics exert less effort in mutual } \\
\text { social control than Protestants }\end{array}$} & Willingness to exert effort in social enforcement & Volunteer index & No & No & - & - & & & & \\
\hline & \multirow{3}{*}{$\begin{array}{l}\text { Different impact of education for Catholics and } \\
\text { Protestants }\end{array}$} & Relationship between Education and: & & & & & & & & \\
\hline & & Religious practice & Yes & Yes & - & - & - & - & - & - \\
\hline & & Trust Church & Yes & No & - & - & & & & \\
\hline \multicolumn{11}{|l|}{ Rule of law: } \\
\hline \multirow{3}{*}{$\begin{array}{l}\text { Catholics support political and legal } \\
\text { institutions less than Protestants }\end{array}$} & Willingness to support political institutions & Tolerance of tax fraud & No & No & + & + & & & & \\
\hline & Willingness to cooperate with the law & Cover up for friends & No & No & + & + & & & & \\
\hline & Confidence in political and legal institutions & Trust institutions & Yes & Yes & - & n. s. & n. s. & n. $s$ & n.s. & - \\
\hline \multicolumn{11}{|l|}{ Homogenous values: } \\
\hline \multirow{2}{*}{$\begin{array}{l}\text { Catholics hold less homogenous values } \\
\text { than Protestants }\end{array}$} & \multirow{2}{*}{$\begin{array}{l}\text { Importance of the family between Catholics and } \\
\text { Protestants }\end{array}$} & Family importance & Yes & Yes & + & + & + & + & + & + \\
\hline & & Trust strangers & Yes & No & - & - & & & & \\
\hline
\end{tabular}

Notes: n. s. = not significance at standard levels.

Sources: Arruñada (2010: 898); and own calculations. 
Table 2: Summary statistics, Arruñada/ISSP 1998 vs. ISSP 2008 (using Calvinist and Broad definitions of Protestantism)

\begin{tabular}{|c|c|c|c|c|c|c|c|c|c|c|c|c|c|c|c|}
\hline \multirow[t]{3}{*}{ Variable } & \multirow{2}{*}{\multicolumn{3}{|c|}{$\begin{array}{c}\text { Arruñada } \\
\text { ISSP } 1998 \\
\end{array}$}} & \multicolumn{6}{|c|}{ Calvinist } & \multicolumn{6}{|c|}{ Broad } \\
\hline & & & & \multicolumn{3}{|c|}{ ISSP 1998} & \multicolumn{3}{|c|}{ ISSP 2008} & \multicolumn{3}{|c|}{ ISSP 1998} & \multicolumn{3}{|c|}{ ISSP 2008} \\
\hline & Obs. & Mean & St. Dev. & Obs. & Mean & St. Dev. & Obs. & Mean & St. Dev. & Obs. & Mean & St. Dev. & Obs. & Mean & St. Dev. \\
\hline \multicolumn{16}{|l|}{ Catholics and Protestants: } \\
\hline Positive working hours & 19,246 & 0.583 & 0.493 & 13,715 & 0.480 & 0.500 & 20,384 & 0.598 & 0.490 & 19,107 & 0.497 & 0.500 & 28,718 & 0.611 & 0.488 \\
\hline Working hours of workers & 9,614 & 40.981 & 13.721 & 6,587 & 41.817 & 13.934 & 12,184 & 40.064 & 14.107 & 9,497 & 41.171 & 13.576 & 17,533 & 39.706 & 13.698 \\
\hline Religious practice & 18,553 & 0.000 & 1.000 & 13,272 & 0.000 & 1.000 & 19,706 & 0.000 & 1.000 & 18,393 & 0.000 & 1.000 & 27,694 & 0.000 & 1.000 \\
\hline Trust institutions & 18,064 & 0.000 & 1.000 & 12,747 & 0.001 & 1.000 & 19,315 & 0.000 & 1.000 & 18,246 & 0.000 & 1.000 & 27,320 & 0.000 & 1.000 \\
\hline Importance of family & 18,158 & 1.503 & 1.209 & 12,828 & 1.423 & 1.218 & 19,137 & 1.435 & 1.284 & 17,964 & 1.490 & 1.214 & 27,129 & 1.477 & 1.274 \\
\hline Catholic & 19,246 & 0.653 & 0.476 & 13,715 & 0.912 & 0.284 & 20,384 & 0.938 & 0.241 & 19,107 & 0.654 & 0.476 & 28,718 & 0.666 & 0.472 \\
\hline Faith & 19,246 & 0.000 & 1.000 & 13,715 & 0.000 & 1.000 & 20,384 & 0.000 & 1.000 & 19,107 & 0.000 & 1.000 & 28,718 & 0.000 & 1.000 \\
\hline Religious upbringing & 19,246 & 0.000 & 1.000 & 13,715 & 0.000 & 1.000 & 20,384 & 0.000 & 1.000 & 19,107 & 0.000 & 1.000 & 28,718 & 0.000 & 1.000 \\
\hline Education & 19,246 & 0.000 & 1.000 & 13,715 & 0.000 & 1.000 & 20,384 & 0.000 & 1.000 & 19,107 & 0.000 & 1.000 & 28,718 & 0.000 & 1.000 \\
\hline Women & 19,246 & 0.557 & 0.497 & 13,715 & 0.558 & 0.497 & 20,384 & 1.568 & 0.495 & 19,107 & 0.557 & 0.497 & 28,718 & 1.568 & 0.495 \\
\hline Age & 19,246 & 0.000 & 1.000 & 13,715 & -0.001 & 1.000 & 20,384 & 0.000 & 1.000 & 19,107 & -0.001 & 1.000 & 28,695 & 0.000 & 1.000 \\
\hline Age squared & 19,246 & 0.000 & 1.000 & 13,715 & -0.001 & 1.000 & 20,384 & 0.000 & 1.000 & 19,107 & -0.001 & 1.000 & 28,695 & 0.000 & 1.000 \\
\hline Widowed & 19,246 & 0.093 & 0.291 & 13,715 & 0.095 & 0.293 & 20,384 & 0.093 & 0.291 & 19,107 & 0.096 & 0.294 & 28,718 & 0.086 & 0.281 \\
\hline Divorced \& seperated & 19,246 & 0.065 & 0.247 & 13,715 & 0.058 & 0.234 & 20,384 & 0.090 & 0.286 & 19,107 & 0.066 & 0.248 & 28,718 & 0.092 & 0.289 \\
\hline Single & 19,246 & 0.215 & 0.411 & 13,715 & 0.215 & 0.411 & 20,384 & 0.280 & 0.449 & 19,107 & 0.218 & 0.413 & 28,718 & 0.285 & 0.451 \\
\hline \multicolumn{16}{|l|}{ Protestants: } \\
\hline Positive working hours & 6,685 & 0.658 & 0.474 & 1,212 & 0.448 & 0.497 & 1,262 & 0.616 & 0.486 & 6,604 & 0.523 & 0.500 & 9,581 & 0.638 & 0.480 \\
\hline Working hours of workers & 3,520 & 39.057 & 13.510 & 543 & 37.484 & 15.789 & 778 & 36.814 & 13.611 & 3,453 & 39.360 & 13.182 & 6,117 & 38.614 & 12.826 \\
\hline Religious practice & 6,348 & -0.243 & 1.084 & 1,135 & -0.116 & 1.150 & 1,202 & -0.337 & 1.204 & 6,256 & -0.214 & 1.096 & 9,175 & -0.132 & 1.119 \\
\hline Trust institutions & 6,360 & 0.109 & 0.954 & 1,137 & 0.181 & 0.866 & 1,209 & 0.199 & 0.874 & 6,395 & 0.030 & 0.956 & 9,199 & 0.213 & 0.969 \\
\hline Importance of family & 6,392 & 1.643 & 1.169 & 1,143 & 1.435 & 1.140 & 1,188 & 1.604 & 1.157 & 6,279 & 1.615 & 1.182 & 9,166 & 1.580 & 1.232 \\
\hline Catholic & 6,685 & 0.000 & 0.000 & 1,212 & 0.000 & 0.000 & 1,262 & 0.000 & 0.000 & 6,604 & 0.000 & 0.000 & 9,581 & 0.000 & 0.000 \\
\hline Faith & 6,685 & -0.181 & 1.033 & 1,212 & -0.063 & 1.025 & 1,262 & -0.138 & 0.994 & 6,604 & -0.171 & 1.038 & 9,581 & -0.090 & 1.054 \\
\hline Religious upbringing & 6,685 & -0.498 & 1.070 & 1,212 & -0.366 & 1.101 & 1,262 & -0.455 & 1.195 & 6,604 & -0.508 & 1.051 & 9,581 & -0.433 & 1.083 \\
\hline Education & 6,685 & 0.171 & 0.946 & 1,212 & 0.175 & 0.937 & 1,262 & 0.058 & 1.025 & 6,604 & 0.169 & 0.950 & 9,581 & 0.166 & 1.033 \\
\hline Women & 6,685 & 0.552 & 0.497 & 1,212 & 0.546 & 0.498 & 1,262 & 1.548 & 0.498 & 6,604 & 0.552 & 0.497 & 9,581 & 1.565 & 0.496 \\
\hline Age & 6,685 & 0.055 & 1.000 & 1,212 & 0.111 & 0.975 & 1,262 & 0.215 & 1.005 & 6,604 & 0.023 & 0.998 & 9,573 & 0.042 & 0.979 \\
\hline Age squared & 6,685 & 0.053 & 1.016 & 1,212 & 0.097 & 0.998 & 1,262 & 0.212 & 1.053 & 6,604 & 0.021 & 1.010 & 9,573 & 0.033 & 0.982 \\
\hline Widowed & 6,685 & 0.091 & 0.288 & 1,212 & 0.092 & 0.290 & 1,262 & 0.111 & 0.314 & 6,604 & 0.096 & 0.295 & 9,581 & 0.076 & 0.264 \\
\hline Divorced \& seperated & 6,685 & 0.082 & 0.275 & 1,212 & 0.069 & 0.254 & 1,262 & 0.103 & 0.304 & 6,604 & 0.082 & 0.275 & 9,581 & 0.098 & 0.298 \\
\hline Single & 6,685 & 0.211 & 0.408 & 1,212 & 0.210 & 0.407 & 1,262 & 0.220 & 0.415 & 6,604 & 0.221 & 0.415 & 9,581 & 0.286 & 0.452 \\
\hline \multicolumn{16}{|l|}{ Catholics: } \\
\hline Positive working hours & 12,561 & 0.543 & 0.498 & 12,503 & 0.483 & 0.500 & 19,122 & 0.596 & 0.491 & 12,503 & 0.483 & 0.500 & 19,137 & 0.597 & 0.491 \\
\hline Working hours of workers & 6,094 & 42.093 & 13.720 & 6,044 & 42.206 & 13.690 & 11,406 & 40.286 & 14.113 & 6,044 & 42.206 & 13.690 & 11,416 & 40.290 & 14.109 \\
\hline Religious practice & 12,205 & 0.126 & 0.937 & 12,137 & 0.010 & 0.984 & 18,504 & 0.022 & 0.981 & 12,137 & 0.110 & 0.928 & 18,519 & 0.065 & 0.929 \\
\hline Trust institutions & 11,686 & -0.060 & 1.019 & 11,610 & -0.017 & 1.011 & 18,106 & -0.013 & 1.006 & 11,851 & -0.016 & 1.023 & 18,121 & -0.108 & 0.998 \\
\hline Importance of family & 11,766 & 1.426 & 1.224 & 11,685 & 1.422 & 1.226 & 17,949 & 1.424 & 1.291 & 11,685 & 1.422 & 1.226 & 17,963 & 1.424 & 1.291 \\
\hline Catholic & 12,561 & 1.000 & 0.000 & 12,503 & 1.000 & 0.000 & 19,122 & 1.000 & 0.000 & 12,503 & 1.000 & 0.000 & 19,137 & 1.000 & 0.000 \\
\hline Faith & 12,561 & 0.096 & 0.969 & 12,503 & 0.006 & 0.997 & 19,122 & 0.009 & 1.000 & 12,503 & 0.090 & 0.967 & 19,137 & 0.045 & 0.968 \\
\hline Religious upbringing & 12,561 & 0.265 & 0.849 & 12,503 & 0.035 & 0.983 & 19,122 & 0.030 & 0.978 & 12,503 & 0.268 & 0.858 & 19,137 & 0.217 & 0.879 \\
\hline Education & 12,561 & -0.091 & 1.016 & 12,503 & -0.017 & 1.004 & 19,122 & -0.004 & 0.998 & 12,503 & -0.090 & 1.014 & 19,137 & -0.083 & 0.972 \\
\hline Women & 12,561 & 0.559 & 0.496 & 12,503 & 0.559 & 0.497 & 19,122 & 1.569 & 0.495 & 12,503 & 0.559 & 0.497 & 19,137 & 1.569 & 0.495 \\
\hline Age & 12,561 & -0.029 & 0.999 & 12,503 & -0.012 & 1.001 & 19,122 & -0.014 & 0.998 & 12,503 & -0.014 & 1.000 & 19,122 & -0.021 & 1.009 \\
\hline Age squared & 12,561 & -0.028 & 0.990 & 12,503 & -0.010 & 0.999 & 19,122 & -0.014 & 0.995 & 12,503 & -0.013 & 0.994 & 19,122 & -0.017 & 1.009 \\
\hline Widowed & 12,561 & 0.094 & 0.292 & 12,503 & 0.095 & 0.294 & 19,122 & 0.092 & 0.289 & 12,503 & 0.095 & 0.294 & 19,137 & 0.092 & 0.289 \\
\hline Divorced \& seperated & 12,561 & 0.056 & 0.230 & 12,503 & 0.057 & 0.232 & 19,122 & 0.089 & 0.285 & 12,503 & 0.057 & 0.232 & 19,137 & 0.089 & 0.285 \\
\hline Single & 12,561 & 0.217 & 0.412 & 12,503 & 0.216 & 0.411 & 19,122 & 0.284 & 0.451 & 12,503 & 0.216 & 0.411 & 19,137 & 0.284 & 0.451 \\
\hline
\end{tabular}

Sources: Arruñada (2010: 903-904); and own calculations in Stata using ISSP Research Group (1998), Religion II Survey and ISSP Research Group (2008), Religion III Survey. 
Table 3: Religious determinants of economic values, ISSP 1998 (omitted category: Protestants)

\begin{tabular}{|c|c|c|c|c|c|c|c|c|c|c|c|c|c|c|c|}
\hline \multirow[t]{4}{*}{ Variable } & \multicolumn{6}{|c|}{ Work ethic hypothesis } & \multicolumn{9}{|c|}{ Social ethic hypothesis } \\
\hline & \multirow{2}{*}{\multicolumn{3}{|c|}{$\begin{array}{c}(1) \\
\text { Positive working hours }\end{array}$}} & \multirow{2}{*}{\multicolumn{3}{|c|}{$\begin{array}{c}\text { (2) } \\
\text { Working hours of workers } \\
\end{array}$}} & \multirow{2}{*}{\multicolumn{3}{|c|}{$\begin{array}{c}\text { (3) } \\
\text { Social control: Religious practice }\end{array}$}} & \multirow{2}{*}{\multicolumn{3}{|c|}{$\begin{array}{c}\text { (4) } \\
\text { Rule of law: Trust institutions }\end{array}$}} & \multirow{2}{*}{\multicolumn{3}{|c|}{$\begin{array}{c}\text { (5) } \\
\text { Homogeneous values: Family importance }\end{array}$}} \\
\hline & & & & & & & & & & & & & & & \\
\hline & Arruñada & Calvinist & Broad & Arruñada & Calvinist & Broad & Arruñada & Calvinist & Broad & Arruñada & Calvinist & Broad & Arruñada & Calvinist & Broad \\
\hline \multicolumn{16}{|c|}{ (a) Difference between Catholics and Protestants (regressions with variable effects): } \\
\hline Catholic & $\begin{array}{l}-0.014 \\
(0.035)\end{array}$ & $\begin{array}{l}-0.051 \\
(0.067)\end{array}$ & $\begin{array}{l}-0.008 \\
(0.036)\end{array}$ & $\begin{array}{l}0.033 \\
(0.445)\end{array}$ & $\begin{array}{c}0.430 \\
(0.999)\end{array}$ & $\begin{array}{c}-0.038 \\
(0.462)\end{array}$ & $\begin{array}{l}-0.034^{*} \\
(0.018)\end{array}$ & $\begin{array}{c}-0.054^{*} \\
(0.032)\end{array}$ & $\begin{array}{c}-0.084 * * * \\
(0.019)\end{array}$ & $\begin{array}{l}-0.012 \\
(0.022)\end{array}$ & $\begin{array}{l}-0.017 \\
(0.037)\end{array}$ & $\begin{array}{c}0.003 \\
(0.022)\end{array}$ & $\begin{array}{c}0.111^{* * *} \\
(0.026)\end{array}$ & $\begin{array}{c}0.179^{* * *} \\
(0.043)\end{array}$ & $\begin{array}{c}0.141^{* * *} \\
(0.026)\end{array}$ \\
\hline Faith & $\begin{array}{c}0.067 * * * \\
(0.023)\end{array}$ & $\begin{array}{c}0.003 \\
(0.053)\end{array}$ & $\begin{array}{c}0.019 \\
(0.024)\end{array}$ & $\begin{array}{l}-0.445 * \\
(0.256)\end{array}$ & $\begin{array}{l}-0.413 \\
(0.776)\end{array}$ & $\begin{array}{l}-0.492^{*} \\
(0.267)\end{array}$ & $\begin{array}{c}0.580^{* * *} \\
(0.012)\end{array}$ & $\begin{array}{c}0.597 * * * \\
(0.027)\end{array}$ & $\begin{array}{c}0.571^{* * *} \\
(0.012)\end{array}$ & $\begin{array}{c}0.057 * * * \\
(0.013)\end{array}$ & $\begin{array}{c}0.081^{* * *} \\
(0.031)\end{array}$ & $\begin{array}{l}0.049 * * * \\
(0.014)\end{array}$ & $\begin{array}{c}-0.230 * * * \\
(0.016)\end{array}$ & $\begin{array}{c}-0.282 * * * \\
(0.036)\end{array}$ & $\begin{array}{c}-0.248^{* * *} \\
(0.017)\end{array}$ \\
\hline Religious upbringing & $\begin{array}{l}-0.004 \\
(0.022)\end{array}$ & $\begin{array}{l}-0.055 \\
(0.051)\end{array}$ & $\begin{array}{c}0.010 \\
(0.026)\end{array}$ & $\begin{array}{c}0.035 \\
(0.248)\end{array}$ & $\begin{array}{c}0.004 \\
(0.712)\end{array}$ & $\begin{array}{c}0.027 \\
(0.296)\end{array}$ & $\begin{array}{l}0.248^{* * *} \\
(0.011)\end{array}$ & $\begin{array}{c}0.246^{* * *} \\
(0.025)\end{array}$ & $\begin{array}{c}0.268^{* * *} \\
(0.013)\end{array}$ & $\begin{array}{l}0.045^{* * *} \\
(0.013)\end{array}$ & $\begin{array}{c}0.042 \\
(0.030)\end{array}$ & $\begin{array}{l}0.046 * * * \\
(0.015)\end{array}$ & $\begin{array}{c}-0.069 * * * \\
(0.016)\end{array}$ & $\begin{array}{l}-0.071 * * \\
(0.034)\end{array}$ & $\begin{array}{c}-0.101^{* * *} \\
(0.018)\end{array}$ \\
\hline Education & $\begin{array}{c}0.219^{* * *} \\
(0.023)\end{array}$ & $\begin{array}{l}0.164^{* * *} \\
(0.054)\end{array}$ & $\begin{array}{c}0.206^{* * *} \\
(0.023)\end{array}$ & $\begin{array}{l}0.746 * * * \\
(0.245)\end{array}$ & $\begin{array}{c}0.559 \\
(0.709)\end{array}$ & $\begin{array}{l}1.014^{* * *} \\
(0.249)\end{array}$ & $\begin{array}{c}0.108^{* * *} \\
(0.010)\end{array}$ & $\begin{array}{c}0.133^{* * *} \\
(0.026)\end{array}$ & $\begin{array}{c}0.094^{* * *} \\
(0.011)\end{array}$ & $\begin{array}{c}0.134^{* * *} \\
(0.013)\end{array}$ & $\begin{array}{c}0.097 * * * \\
(0.030)\end{array}$ & $\begin{array}{c}0.114^{* * *} \\
(0.013)\end{array}$ & $\begin{array}{c}-0.075 * * * \\
(0.015)\end{array}$ & $\begin{array}{l}-0.035 \\
(0.034)\end{array}$ & $\begin{array}{c}-0.081^{* * * *} \\
(0.015)\end{array}$ \\
\hline Catholic $\times$ Faith & $\begin{array}{c}-0.131 * * * \\
(0.027)\end{array}$ & $\begin{array}{c}-0.055 \\
(0.055)\end{array}$ & $\begin{array}{c}-0.081 * * * \\
(0.027)\end{array}$ & $\begin{array}{c}0.649 * * \\
(0.316)\end{array}$ & $\begin{array}{c}0.734 \\
(0.801)\end{array}$ & $\begin{array}{c}0.701^{* *} \\
(0.324)\end{array}$ & $\begin{array}{c}-0.167 * * * \\
(0.014)\end{array}$ & $\begin{array}{c}-0.199 * * * \\
(0.028)\end{array}$ & $\begin{array}{c}-0.169 * * * \\
(0.014)\end{array}$ & $\begin{array}{c}0.048^{* * *} \\
(0.017)\end{array}$ & $\begin{array}{c}0.021 \\
(0.032)\end{array}$ & $\begin{array}{c}0.039 * * \\
(0.017)\end{array}$ & $\begin{array}{c}0.121^{* * *} \\
(0.02)\end{array}$ & $\begin{array}{c}0.186 * * * \\
(0.037)\end{array}$ & $\begin{array}{c}0.143 * * * \\
(0.021)\end{array}$ \\
\hline $\begin{array}{l}\text { CatholicR } \times \text { Religious } \\
\text { upbringing }\end{array}$ & $\begin{array}{l}-0.040 \\
(0.028)\end{array}$ & $\begin{array}{c}0.028 \\
(0.053)\end{array}$ & $\begin{array}{l}-0.005 * \\
(0.003)\end{array}$ & $\begin{array}{l}-0.374 \\
(0.32)\end{array}$ & $\begin{array}{l}-0.465 \\
(0.740)\end{array}$ & $\begin{array}{l}-0.038 \\
(0.036)\end{array}$ & $\begin{array}{c}0.048 * * * \\
(0.014)\end{array}$ & $\begin{array}{c}0.013 \\
(0.026)\end{array}$ & $\begin{array}{c}0.001 \\
(0.002)\end{array}$ & $\begin{array}{c}0.011 \\
(0.018)\end{array}$ & $\begin{array}{c}0.013 \\
(0.032)\end{array}$ & $\begin{array}{l}-0.001 \\
(0.002)\end{array}$ & $\begin{array}{c}0.060^{* * *} \\
(0.021)\end{array}$ & $\begin{array}{c}0.072 * * \\
(0.036)\end{array}$ & $\begin{array}{c}0.010^{* * *} \\
(0.002)\end{array}$ \\
\hline Catholic $\times$ Education & $\begin{array}{c}-0.035 \\
(0.028)\end{array}$ & $\begin{array}{c}0.029 \\
(0.056)\end{array}$ & $\begin{array}{c}-0.014 \\
(0.027)\end{array}$ & $\begin{array}{c}-0.821 * * * \\
(0.312)\end{array}$ & $\begin{array}{c}-0.611 \\
(0.734)\end{array}$ & $\begin{array}{c}-1.092^{* * *} \\
(0.312)\end{array}$ & $\begin{array}{c}-0.091 * * * \\
(0.012)\end{array}$ & $\begin{array}{c}-0.114^{* * *} \\
(0.027)\end{array}$ & $\begin{array}{c}-0.078^{* * *} \\
(0.013)\end{array}$ & $\begin{array}{c}-0.082 * * * \\
(0.016)\end{array}$ & $\begin{array}{l}-0.054^{*} \\
(0.032)\end{array}$ & $\begin{array}{c}-0.060^{* * *} \\
(0.016)\end{array}$ & $\begin{array}{c}0.104 * * * \\
(0.018)\end{array}$ & $\begin{array}{l}0.058^{*} \\
(0.035)\end{array}$ & $\begin{array}{c}0.118^{* * *} \\
(0.018)\end{array}$ \\
\hline Observations & 18,233 & 11,684 & 17,139 & 9,614 & 5,776 & 9,026 & 18,553 & 11,860 & 17,544 & 18,046 & 11,415 & 17,371 & 18,158 & 11,432 & 17,103 \\
\hline \multicolumn{16}{|c|}{ (b) Difference between Catholics and Protestants (regressions without variable effects): } \\
\hline Catholic & $\begin{array}{c}-0.03 \\
(0.035)\end{array}$ & $\begin{array}{l}-0.052 \\
(0.060)\end{array}$ & $\begin{array}{l}-0.013 \\
(0.034)\end{array}$ & $\begin{array}{l}-0.192 \\
(0.428)\end{array}$ & $\begin{array}{c}-0.05 \\
(0.767)\end{array}$ & $\begin{array}{c}-0.223 \\
(0.413)\end{array}$ & $\begin{array}{c}0.078 * * * \\
(0.022)\end{array}$ & $\begin{array}{c}0.092 * * \\
(0.038)\end{array}$ & $\begin{array}{c}0.017 \\
(0.022)\end{array}$ & $\begin{array}{c}0.007 \\
(0.002)\end{array}$ & $\begin{array}{c}-0.002 \\
(0.033)\end{array}$ & $\begin{array}{c}0.024 \\
(0.021)\end{array}$ & $\begin{array}{c}0.110^{* * *} \\
(0.025)\end{array}$ & $\begin{array}{c}0.153^{* * *} \\
(0.039)\end{array}$ & $\begin{array}{c}0.156 * * * \\
(0.024)\end{array}$ \\
\hline Observations & 18,233 & 13,080 & 17,988 & 9,614 & 6,587 & 9,497 & 18,553 & 13,272 & 18,393 & 18,046 & 12,747 & 18,246 & 18,158 & 12,828 & 17,964 \\
\hline
\end{tabular}

Sources: Arruñada (2010: 905); and own calculations in Stata using ISSP Research Group (1998), Religion II Survey

Notes: All models (in columns) estimated with constants and demographic and country controls, whose coefficients are not reported in the table. Equation (1), probit; equations (2) and (4), OLS; equations (3) and (5), ordered probit. *, **, *** Significance at 10, 5, and 1\% levels. Robust standard errors in parentheses. 
Table 4: Religious determinants of economic values, ISSP 1998 vs. ISSP 2008 (using Calvinist definition of Protestantism)

\begin{tabular}{|c|c|c|c|c|c|c|c|c|c|c|}
\hline \multirow[t]{4}{*}{ Variable } & \multicolumn{4}{|c|}{ Work ethic hypothesis } & \multicolumn{6}{|c|}{ Social ethic hypothesis } \\
\hline & \multirow{2}{*}{\multicolumn{2}{|c|}{$\begin{array}{c}\text { (6) } \\
\text { Positive working hours } \\
\end{array}$}} & \multirow{2}{*}{\multicolumn{2}{|c|}{$\begin{array}{c}\text { (7) } \\
\text { Working hours of workers }\end{array}$}} & \multirow{2}{*}{\multicolumn{2}{|c|}{$\begin{array}{c}\text { (8) } \\
\text { Religious practice }\end{array}$}} & \multirow{2}{*}{\multicolumn{2}{|c|}{$\begin{array}{c}\text { (9) } \\
\text { Trust institutions } \\
\end{array}$}} & \multirow{2}{*}{\multicolumn{2}{|c|}{$\begin{array}{c}(10) \\
\text { Family importance }\end{array}$}} \\
\hline & & & & & & & & & & \\
\hline & ISSP 1998 & ISSP 2008 & ISSP 1998 & ISSP 2008 & ISSP 1998 & ISSP 2008 & ISSP 1998 & ISSP 2008 & ISSP 1998 & ISSP 2008 \\
\hline \multicolumn{11}{|c|}{ (a) Difference between Catholics and Protestants (regressions with variable effects): } \\
\hline Catholic & $\begin{array}{l}-0.051 \\
(0.067)\end{array}$ & $\begin{array}{c}0.088 \\
(0.080)\end{array}$ & $\begin{array}{c}0.430 \\
(0.999)\end{array}$ & $\begin{array}{c}1.184 \\
(0.825)\end{array}$ & $\begin{array}{l}-0.054^{*} \\
(0.032)\end{array}$ & $\begin{array}{c}0.146 * * * \\
(0.046)\end{array}$ & $\begin{array}{l}-0.017 \\
(0.037)\end{array}$ & $\begin{array}{l}-0.032 \\
(0.048)\end{array}$ & $\begin{array}{c}0.179 * * * \\
(0.043)\end{array}$ & $\begin{array}{l}0.130 * * \\
(0.055)\end{array}$ \\
\hline Faith & $\begin{array}{c}0.003 \\
(0.053)\end{array}$ & $\begin{array}{c}0.041 \\
(0.066)\end{array}$ & $\begin{array}{l}-0.413 \\
(0.776)\end{array}$ & $\begin{array}{l}-1.560^{* *} \\
(0.754)\end{array}$ & $\begin{array}{c}0.597 * * * \\
(0.027)\end{array}$ & $\begin{array}{c}0.548 * * * \\
(0.040)\end{array}$ & $\begin{array}{c}0.081 * * * \\
(0.031)\end{array}$ & $\begin{array}{c}0.021 \\
(0.043)\end{array}$ & $\begin{array}{c}-0.282^{* * *} \\
(0.036)\end{array}$ & $\begin{array}{c}-0.244^{* * *} \\
(0.049)\end{array}$ \\
\hline Religious upbringing & $\begin{array}{l}-0.055 \\
(0.051)\end{array}$ & $\begin{array}{l}-0.154^{* *} \\
(0.065)\end{array}$ & $\begin{array}{c}0.004 \\
(0.712)\end{array}$ & $\begin{array}{c}0.122 \\
(0.659)\end{array}$ & $\begin{array}{c}0.246^{* * *} \\
(0.025)\end{array}$ & $\begin{array}{c}0.267 * * * \\
(0.035)\end{array}$ & $\begin{array}{c}0.042 \\
(0.030)\end{array}$ & $\begin{array}{l}0.081^{*} \\
(0.042)\end{array}$ & $\begin{array}{c}-0.071^{* *} \\
(0.034)\end{array}$ & $\begin{array}{c}0.013 \\
(0.049)\end{array}$ \\
\hline Education & $\begin{array}{c}0.164 * * * \\
(0.054)\end{array}$ & $\begin{array}{c}0.053 \\
(0.063)\end{array}$ & $\begin{array}{c}0.559 \\
(0.709)\end{array}$ & $\begin{array}{c}0.887 \\
(0.628)\end{array}$ & $\begin{array}{c}0.133^{* * *} \\
(0.026)\end{array}$ & $\begin{array}{c}0.075 * * \\
(0.036)\end{array}$ & $\begin{array}{c}0.097 * * * \\
(0.030)\end{array}$ & $\begin{array}{c}0.053 \\
(0.034)\end{array}$ & $\begin{array}{l}-0.035 \\
(0.034)\end{array}$ & $\begin{array}{l}0.069 * \\
(0.040)\end{array}$ \\
\hline Catholic $\times$ Faith & $\begin{array}{l}-0.055 \\
(0.055)\end{array}$ & $\begin{array}{l}-0.051 \\
(0.067)\end{array}$ & $\begin{array}{c}0.734 \\
(0.801)\end{array}$ & $\begin{array}{l}1.342^{*} \\
(0.761)\end{array}$ & $\begin{array}{c}-0.199 * * * \\
(0.028)\end{array}$ & $\begin{array}{c}-0.159 * * * \\
(0.040)\end{array}$ & $\begin{array}{c}0.021 \\
(0.032)\end{array}$ & $\begin{array}{c}0.046 \\
(0.044)\end{array}$ & $\begin{array}{c}0.186 * * * \\
(0.037)\end{array}$ & $\begin{array}{c}0.124 * * \\
(0.050)\end{array}$ \\
\hline $\begin{array}{l}\text { CatholicR } \times \text { Religious } \\
\text { upbringing }\end{array}$ & $\begin{array}{c}0.028 \\
(0.053)\end{array}$ & $\begin{array}{c}0.107 \\
(0.066)\end{array}$ & $\begin{array}{l}-0.465 \\
(0.740)\end{array}$ & $\begin{array}{l}-0.038 \\
(0.671)\end{array}$ & $\begin{array}{c}0.013 \\
(0.026)\end{array}$ & $\begin{array}{c}0.036 \\
(0.036)\end{array}$ & $\begin{array}{c}0.013 \\
(0.032)\end{array}$ & $\begin{array}{c}-0.090^{* *} \\
(0.042)\end{array}$ & $\begin{array}{l}0.072 * * \\
(0.036)\end{array}$ & $\begin{array}{l}-0.006 \\
(0.050)\end{array}$ \\
\hline Catholic $\mathrm{x}$ Education & $\begin{array}{c}0.029 \\
(0.056)\end{array}$ & $\begin{array}{c}0.128^{* *} \\
(0.063)\end{array}$ & $\begin{array}{l}-0.611 \\
(0.734)\end{array}$ & $\begin{array}{l}-0.855 \\
(0.639)\end{array}$ & $\begin{array}{c}-0.114^{* * *} \\
(0.027)\end{array}$ & $\begin{array}{c}-0.099 * * * \\
(0.036)\end{array}$ & $\begin{array}{l}-0.054 * \\
(0.032)\end{array}$ & $\begin{array}{l}-0.022 \\
(0.035)\end{array}$ & $\begin{array}{l}0.058^{*} \\
(0.035)\end{array}$ & $\begin{array}{c}0.011 \\
(0.041)\end{array}$ \\
\hline Observations & 11,684 & 18,865 & 5,776 & 11,325 & 11,860 & 18,258 & 11,415 & 17,876 & 11,432 & 17,690 \\
\hline \multicolumn{11}{|c|}{ (b) Difference between Catholics and Protestants (regressions without variable effects): } \\
\hline Catholic & $\begin{array}{l}-0.052 \\
(0.060)\end{array}$ & $\begin{array}{l}0.083^{*} \\
(0.051)\end{array}$ & $\begin{array}{c}-0.05 \\
(0.767)\end{array}$ & $\begin{array}{c}0.393 \\
(0.537)\end{array}$ & $\begin{array}{l}0.092 * * \\
(0.038)\end{array}$ & $\begin{array}{c}0.118^{* * *} \\
(0.038)\end{array}$ & $\begin{array}{l}-0.002 \\
(0.033)\end{array}$ & $\begin{array}{c}0.032 \\
(0.030)\end{array}$ & $\begin{array}{c}0.153 * * * \\
(0.039)\end{array}$ & $\begin{array}{c}0.135^{* * *} \\
(0.037)\end{array}$ \\
\hline Observations & 13,080 & 20,384 & 6,587 & 12,184 & 13,272 & 19,706 & 12,747 & 19,315 & 12,828 & 19,137 \\
\hline
\end{tabular}

Sources: Own calculations in Stata using ISSP Research Group (1998), Religion II Survey; and ISSP Research Group (2008), Religion III Survey.

Notes: All models (in columns) estimated with constants and demographic and country controls, whose coefficients are not reported in the table. Equation (6), probit; equations (7) and (9), OLS; equations (8) and (10), ordered probit. *, **, *** Significance at 10,5 , and $1 \%$ levels. Robust standard errors in parentheses. 
Table 5: Religious determinants of economic values, ISSP 1998 vs. ISSP 2008 (using broad definition of Protestantism)

\begin{tabular}{|c|c|c|c|c|c|c|c|c|c|c|}
\hline \multirow[t]{4}{*}{ Variable } & \multicolumn{4}{|c|}{ Work ethic hypothesis } & \multicolumn{6}{|c|}{ Social ethic hypothesis } \\
\hline & \multirow{2}{*}{\multicolumn{2}{|c|}{$\begin{array}{c}\text { (11) } \\
\text { Positive working hours }\end{array}$}} & \multirow{2}{*}{\multicolumn{2}{|c|}{$\begin{array}{c}\text { (12) } \\
\text { Working hours of workers }\end{array}$}} & \multirow{2}{*}{\multicolumn{2}{|c|}{$\begin{array}{c}\text { (13) } \\
\text { Religious practice }\end{array}$}} & \multirow{2}{*}{\multicolumn{2}{|c|}{$\begin{array}{c}\text { (14) } \\
\text { Trust institutions } \\
\end{array}$}} & \multirow{2}{*}{\multicolumn{2}{|c|}{$\begin{array}{c}\text { (15) } \\
\text { Family importance }\end{array}$}} \\
\hline & & & & & & & & & & \\
\hline & ISSP 1998 & ISSP 2008 & ISSP 1998 & ISSP 2008 & ISSP 1998 & ISSP 2008 & ISSP 1998 & ISSP 2008 & ISSP 1998 & ISSP 2008 \\
\hline \multicolumn{11}{|c|}{ (a) Difference between Catholics and Protestants (regressions with variable effects): } \\
\hline \multirow[t]{2}{*}{ Catholic } & -0.008 & 0.036 & -0.038 & -0.372 & $-0.084 * * *$ & $-0.127 * * *$ & 0.003 & $-0.159 * * *$ & $0.155^{* * *}$ & $0.118^{* * *}$ \\
\hline & $(0.036)$ & $(0.023)$ & $(0.462)$ & $(0.311)$ & $(0.019)$ & $(0.013)$ & $(0.022)$ & $(0.017)$ & $(0.027)$ & $(0.019)$ \\
\hline \multirow[t]{2}{*}{ Faith } & 0.019 & 0.000 & $-0.492 *$ & $-0.380^{* *}$ & $0.571^{* * *}$ & $0.529 * * *$ & $0.049 * * *$ & $0.021 *$ & $-0.247 * * *$ & $-0.216^{* * *}$ \\
\hline & $(0.024)$ & $(0.018)$ & $(0.267)$ & $(0.193)$ & $(0.012)$ & $(0.010)$ & $(0.014)$ & $(0.011)$ & $(0.017)$ & $(0.013)$ \\
\hline \multirow[t]{2}{*}{ Religious upbringing } & 0.010 & $-0.071 * * *$ & 0.027 & 0.134 & $0.268^{* * *}$ & $0.290^{* * *}$ & $0.046^{* * *}$ & $-0.024^{* *}$ & $-0.100^{* * *}$ & $-0.149^{* * *}$ \\
\hline & $(0.026)$ & $(0.018)$ & $(0.296)$ & $(0.197)$ & $(0.013)$ & $(0.010)$ & $(0.015)$ & $(0.012)$ & $(0.018)$ & $(0.014)$ \\
\hline \multirow[t]{2}{*}{ Education } & $0.206 * * *$ & $0.206 * * *$ & $1.014 * * *$ & $0.858 * * *$ & $0.094^{* * *}$ & $0.057 * * *$ & $0.114 * * *$ & $0.095 * * *$ & $-0.078 * * *$ & 0.010 \\
\hline & $(0.023)$ & $(0.016)$ & $(0.249)$ & $(0.189)$ & $(0.011)$ & $(0.009)$ & $(0.013)$ & $(0.010)$ & $(0.016)$ & $(0.011)$ \\
\hline \multirow[t]{2}{*}{ Catholic $\times$ Faith } & $-0.081^{* * *}$ & -0.014 & $0.701^{* *}$ & 0.265 & $-0.169 * * *$ & $-0.141^{* * *}$ & $0.039 * *$ & $0.052 * * *$ & $0.141^{* * *}$ & $0.085 * * *$ \\
\hline & $(0.027)$ & $(0.021)$ & $(0.324)$ & $(0.234)$ & $(0.014)$ & $(0.011)$ & $(0.017)$ & $(0.014)$ & $(0.021)$ & $(0.016)$ \\
\hline \multirow{2}{*}{$\begin{array}{l}\text { CatholicR } \times \text { Religious } \\
\text { upbringing }\end{array}$} & $-0.005^{*}$ & 0.035 & -0.038 & -0.005 & 0.001 & -0.006 & -0.001 & 0.021 & $0.101^{* * *}$ & $0.149^{* * *}$ \\
\hline & $(0.003)$ & $(0.021)$ & $(0.036)$ & $(0.230)$ & $(0.002)$ & $(0.012)$ & $(0.002)$ & $(0.014)$ & $(0.022)$ & $(0.016)$ \\
\hline \multirow[t]{2}{*}{ Catholic $\times$ Education } & -0.014 & -0.024 & $-1.092^{* * *}$ & $-0.851^{* * *}$ & $-0.078^{* * *}$ & $-0.071^{* * *}$ & $-0.060 * * *$ & $-0.056^{* * *}$ & $0.112 * * *$ & $0.077^{* * *}$ \\
\hline & $(0.027)$ & $(0.019)$ & $(0.312)$ & $(0.234)$ & $(0.013)$ & $(0.010)$ & $(0.016)$ & $(0.013)$ & $(0.019)$ & $(0.014)$ \\
\hline Observations & 17,139 & 27,932 & 9,026 & 17,176 & 17,544 & 26,936 & 17,371 & 26,587 & 16,897 & 26,383 \\
\hline \multicolumn{11}{|c|}{ (b) Difference between Catholics and Protestants (regressions without variable effects): } \\
\hline \multirow[t]{2}{*}{ Catholic } & -0.013 & 0.014 & -0.223 & $-0.517^{*}$ & 0.017 & $-0.101^{* * *}$ & 0.024 & $-0.170^{* * *}$ & $0.156^{* * *}$ & $0.139 * * *$ \\
\hline & $(0.034)$ & -0.022 & $(0.413)$ & $(0.293)$ & $(0.022)$ & $(0.016)$ & $(0.021)$ & $(0.016)$ & $(0.024)$ & $(0.018)$ \\
\hline Observations & 17,988 & 28,695 & 9,497 & 17,522 & 18,393 & 27,672 & 18,246 & 27,297 & 17,964 & 27,108 \\
\hline
\end{tabular}

Sources: Own calculations in Stata using ISSP Research Group (1998), Religion II Survey; and ISSP Research Group (2008), Religion III Survey.

Notes: All models (in columns) estimated with constants and demographic and country controls, whose coefficients are not reported in the table. Equation (11), probit; equations (12) and (14), OLS; equations (13) and (15), ordered probit. *,**, *** Significance at 10,5 , and $1 \%$ levels. Robust standard errors in parentheses. 
Table S1: Variable definitions, Arruñada/ISSP 1998 vs. ISSP 2008

\begin{tabular}{|c|c|c|c|c|c|}
\hline \multirow[t]{2}{*}{ Variable } & \multicolumn{2}{|r|}{ Arruñada/ISSP 1998} & \multicolumn{2}{|r|}{ ISSP 2008} & \multirow[t]{2}{*}{ Value of variable } \\
\hline & Code & Survey question & Code & Survey question & \\
\hline \multicolumn{6}{|l|}{ Work ethic: } \\
\hline Positive working hours & $v 213$ & Hours worked weekly & WRKHRS & R: Hours worked weekly & Binary variable (equals 1 if hours > 0 ) \\
\hline Working hours of workers & $v 213$ & Hours worked weekly & WRKHRS & R: Hours worked weekly & Hours worked (observation ommitted if hours $=0$ ) \\
\hline \multicolumn{6}{|l|}{ Social control: } \\
\hline \multirow[t]{5}{*}{ Religious practice } & Index & Built with scores of first principal components from v58,v59,v60 and v218 & Index & Built with scores of first principal components from V59,V60,V63 and ATTEND & Standardised \\
\hline & v58 & How often do you pray? & v59 & How often do you pray? & Frequency \\
\hline & v59 & How often take part in church activities? & v60 & R: Attendance of religious services & Frequency \\
\hline & v60 & Would you describe yourself as extremely religious? & V63 & $R$ describes self as religious & Recoded for the variable to increase with religiosity \\
\hline & $\mathrm{v} 218$ & How often do you attend religious services? & ATTEND & Attendance of religious services & Frequency \\
\hline \multicolumn{6}{|l|}{ Rule of law: } \\
\hline \multirow[t]{3}{*}{ Trust institutions } & Index & Built with scores of first principal components from v20 and v23 & Index & Built with scores of first principal components fromV14 and V17 & Standardised \\
\hline & $v 20$ & How much confidence respondent has in parliament? & V14 & How much confidence respondent has in parliament? & Variable to increase with confidence \\
\hline & $v 21$ & How much confidence respondent has in courts and the legal system? & V17 & How much confidence respondent has in courts and the legal system? & Variable to increase with confidence \\
\hline \multicolumn{6}{|l|}{ Homogenous values: } \\
\hline \multirow[t]{3}{*}{ Importance of family } & Index & Built as difference between v7 and v8 & Index & Built as difference between V7 and V8 & Difference \\
\hline & $v 7$ & Do you think it is wrong to have sexual relations before marriage? & $V 7$ & Do you think it is wrong to have sexual relations before marriage? & Tolerance \\
\hline & $v 8$ & Do you think it is wrong to have sexual relations with others than spouse? & V8 & Do you think it is wrong to have sexual relations with others than spouse? & Tolerance \\
\hline \multicolumn{6}{|l|}{ Independent variables: } \\
\hline Catholic & $v 217$ & R: Religious denomination & RELIG & R: Religious denomination & Binary variable \\
\hline CatholicR & v53 & Religion $\mathrm{R}$ was raised in & v52 & What religion, if any, were you raised in? & Binary variable \\
\hline \multirow[t]{4}{*}{ Faith } & Index & Built with scores of first principal components from v39 to v41 & Index & Built with scores of first principal components from V35 to V37 & Standardised \\
\hline & v39 & $\mathrm{R}$ believe in life after death? & V35 & Belief in life after death & Variable to increase with strength of belief \\
\hline & $v 40$ & R believe in Heaven? & V36 & Belief in heaven & Binary variable \\
\hline & $v 41$ & $\mathrm{R}$ believe in Hell? & V37 & Belief in hell & Binary variable \\
\hline Religious upbringing & v57 & $\mathrm{R}$ age 11-12 yrs, how often attend church & v58 & $\mathrm{R}$ age $11-12, \mathrm{R}$ attend church & Frequency \\
\hline Education & $v 205$ & R: Education II: Highest education level & DEGREE & R: Education II-highest education level & Standardised \\
\hline \multicolumn{6}{|l|}{ Control variables: } \\
\hline Sex & $v 200$ & Sex of respondent & SEX & Sex of respondent & Binary variable \\
\hline Marital status & $v 202$ & R: Marital status & MARITAL & R: Marital status & Binary variable \\
\hline Age & $v 201$ & Age of respondent & $A G E$ & Age of respondant & Standardised \\
\hline Country & v3 & Country & V4 & Country & Standardised \\
\hline
\end{tabular}

Sources: Arruñada (2010: Appendix); ISSP 1998 Codebook; and ISSP 2008 Codebook. 
Table S2: Comparison of means, ISSP 1998 vs. ISSP 2008

\begin{tabular}{|c|c|c|c|c|c|c|}
\hline \multirow[t]{3}{*}{ Variable } & \multicolumn{6}{|c|}{ Difference (ISSP 2008 - ISSP 1998) } \\
\hline & \multicolumn{2}{|c|}{ Catholics and Protestants } & \multicolumn{2}{|c|}{ Protestants } & \multicolumn{2}{|c|}{ Catholics } \\
\hline & Calvinist & Broad & Calvinist & Broad & Calvinist & Broad \\
\hline Positive working hours & $0.117 * * *$ & $0.113^{* * *}$ & $0.168 * * *$ & $0.116^{* * *}$ & $0.113^{* * *}$ & $0.113^{* * *}$ \\
\hline Working hours of workers & $-1.753 * * *$ & $-1.466 * * *$ & $-0.671 * * *$ & $-0.746^{* * *}$ & -1.920 & $-1.916^{* * *}$ \\
\hline Importance of family & 0.012 & $-0.013^{* * *}$ & 0.170 & -0.035 & $0.002 * * *$ & 0.002 \\
\hline Catholic & $0.026 * * *$ & $0.012 * * *$ & & & & \\
\hline Women & 1.010 & $1.011 * * *$ & 1.002 & 1.013 & 1.010 & 1.010 \\
\hline Widowed & -0.002 & $-0.009 * * *$ & 0.019 & -0.020 & -0.004 & $-0.004 * * *$ \\
\hline Divorced \& seperated & $0.032 * * *$ & $0.026^{* * *}$ & $0.034 * * *$ & $0.016^{* * *}$ & $0.032 * * *$ & $0.032 * * *$ \\
\hline Single & $0.065 * * *$ & $0.067 * * *$ & $-0.011 * * *$ & $0.066 * * *$ & 0.068 & $0.068 * * *$ \\
\hline
\end{tabular}

Sources: Own calculations in Stata using ISSP Research Group (1998), Religion II Survey; and ISSP Research Group (2008), Religion III Survey.

Notes: Difference in means is calculated by subtracting the 1998 form the 2008 means. *, **, *** Significance at 10, 5, and 1\% levels in a two-tailed t-test following Levene's (1960) test for equality of variances. 
Table S3: Comparison of residual heterogeneity, Catholics vs. Protestants

\begin{tabular}{|c|c|c|c|c|c|}
\hline \multirow[t]{3}{*}{ Variable } & \multicolumn{5}{|c|}{ Difference (Protestants - Catholics) } \\
\hline & \multirow[t]{2}{*}{ Arruñada } & \multicolumn{2}{|c|}{ ISSP 1998} & \multicolumn{2}{|c|}{ ISSP 2008} \\
\hline & & Calvinist & Broad & Calvinist & Broad \\
\hline Positive working hours & $-0.039 * * *$ & $0.015 * *$ & $-0.015 * * *$ & $0.017 * * *$ & $0.012 * * *$ \\
\hline Working hours of workers & $-1.701 * * *$ & $1.035 * * *$ & $-1.021 * * *$ & $-0.888 * * *$ & $-1.245 * * *$ \\
\hline Religious practice & 0.022 & $0.136^{* * *}$ & 0.027 & $0.215 * * *$ & $0.105 * * *$ \\
\hline Trust institutions & $-0.050 * * *$ & $-0.097 * * *$ & $-0.055 * * *$ & $-0.050 * * *$ & $-0.060 * * *$ \\
\hline Family importance & $-0.076^{* * *}$ & $-0.002 * * *$ & $0.000 * * *$ & $-0.001 * * *$ & $0.000 * * *$ \\
\hline
\end{tabular}

Sources: Arruñada (2010: 906), and own calculations in Stata using OLS regressions with the same independent variables as in Panels (a) of Tables 4, 5 and 6.

Notes: *** Significance at $1 \%$, using Levene's (1960) test for equality of variances. 